\title{
Synthesis of 2-Azidomethyl-5-ethynylfuran: A New Bio-Derived Self-Clickable Building Block
}

\author{
Bogdan Ya. Karlinskii \\ Leonid V. Romashov \\ Konstantin I. Galkin \\ Pavel G. Kislitsyn \\ Valentine P. Ananikov* (D)
}

N. D. Zelinsky Institute of Organic Chemistry of the Russian Academy of Sciences, Leninsky prospect 47, Moscow, 119991. Russian Federation

val@ioc.ac.ru

Published as part of the 50 Years SYNTHESIS - Golden Anniversary Issue

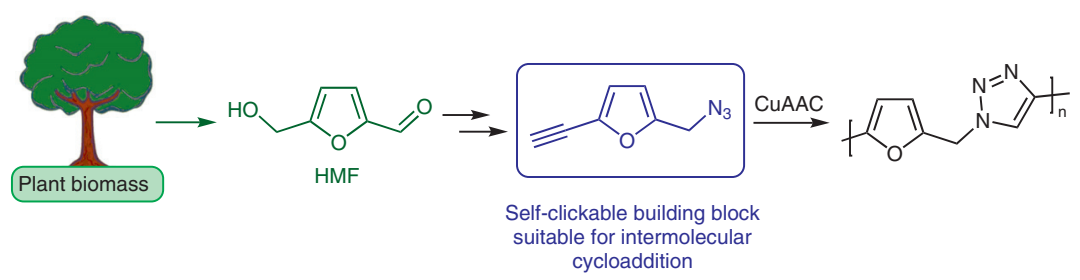

\author{
Received: 05.10.2018 \\ Accepted after revision: 26.11.2018 \\ Published online: 08.01 .2019 \\ DOI: 10.1055/s-0037-1610414; Art ID: ss-2018-z0676-op \\ License terms: CCO
}

Abstract 2-Azidomethyl-5-ethynylfuran, a new ambivalent compound with both azide and alkyne moieties that can be used as a self-clickable monomer, is synthesized starting directly from renewable biomass. The reactivity of the azide group linked to furfural is tested via the efficient preparation of a broad range of furfural-containing triazoles in good to excellent yields using a 'green' copper(I)-catalyzed azide-alkyne cycloaddition procedure. Access to new bio-based chemicals and oligomeric materials via a click-chemistry approach is also demonstrated using this bio-derived building block.

Key words 5-(hydroxymethyl)furfural, click chemistry, biomass conversion, triazoles, oligomers

The production and conversion of bio-derived platform chemicals and building blocks has received significant attention in order to meet the demands of sustainable development and green chemistry concepts. ${ }^{1}$ 5-(Hydroxymethyl)furfural (HMF), produced by catalytic dehydration of hexose carbohydrates, has been recently nominated as a 'sleeping giant' 2 of sustainable chemistry, possessing numerous applications especially in the production of biofuels, ${ }^{3}$ polymers ${ }^{4}$ and fine chemicals. ${ }^{5}$ However, most of the described HMF derivatives were afforded by various redox processes and therefore comprise one or two oxygen-containing functional groups (Scheme 1, Types 1 and 2), whereas literature reports on bio-based furans with two oxygen-free functional groups (Scheme 1, Type 3 ) are less prominent. Furthermore, the vast majority of HMF-based materials are either polyesters or polyamides. ${ }^{4}$ Examples of HMF-based polymers with other types of backbone remain quite rare.

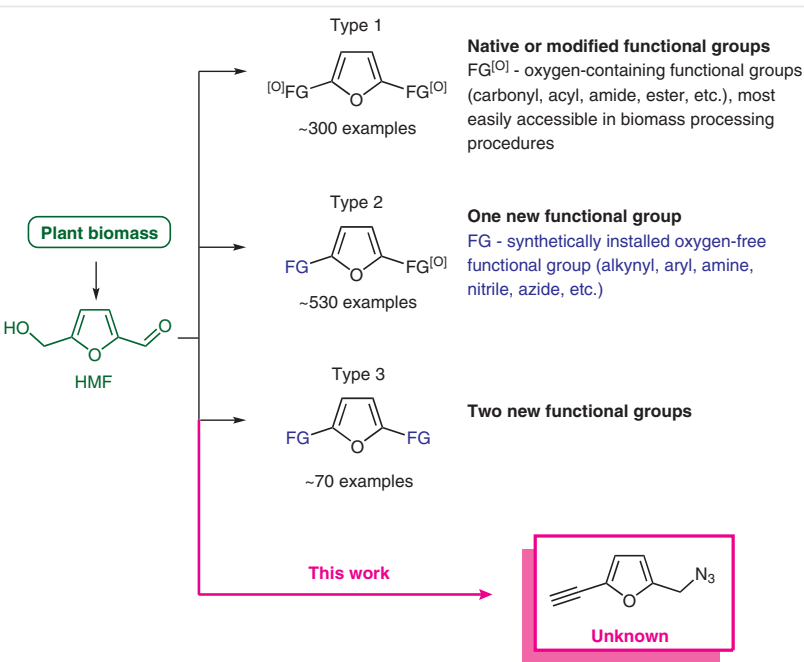

Scheme 1 Three types of HMF derivatives (according to a literature search; ${ }^{6}$ for representative examples see the reviews ${ }^{1 \mathrm{a}, \mathrm{b}}$ )

The introduction of two oxygen-free functional groups into bio-derived sustainable building blocks is currently one of the key research directions. This problem is especially challenging for the cases of two mutually reactive functional groups, for example, azide and alkyne fragments that are able to quickly combine into a triazole via a Huisgen dipolar cycloaddition. ${ }^{7}$ A catalytic modification of this reaction, the copper(I)-catalyzed azide-alkyne cycloaddition (CuAAC), has proved to be one of the most important linking reactions for click-chemistry applications. ${ }^{8}$ It usually proceeds under mild conditions with high yields and regioselectivity and has numerous advantages for use in materials science, ${ }^{9}$ drug development ${ }^{10}$ and other fields of organic, bioorganic and medicinal chemistry. ${ }^{11}$ 
Indeed, among a small number of HMF derivatives with two oxygen-free functional groups there is only one example each of the synthesis of alkyne- or azide-containing furanyls: 2,5-diethynylfuran ${ }^{12}$ and 2,5-bis(azidomethyl)furan, ${ }^{13}$ but there are no examples of combining these moieties in one molecule. More generally, only a few azide ${ }^{14}$ and alkyne ${ }^{15}$ derivatives have been synthesized from biomass-derived furans, despite their unquestionable relevance for the easy and rapid 'click' synthesis of triazoles, ${ }^{16}$ which are themselves reported to have antitubercular ${ }^{17}$ and anticancer ${ }^{18}$ activity.

In this work, we report a synthetic route to the new, 'self-clickable' building block, 2-azidomethyl-5-ethynylfuran (AMEF) (6). The term 'self-clickable' describes an ambivalent reactivity and ability to engage in intermolecular cycloaddition related to the presence of both azide and alkyne moieties. This duality allows 'click' polycondensation ${ }^{19}$ to be carried out under mild conditions leading to heterocyclic furan-triazole oligomers. In addition, a number of new biobased triazoles were obtained via a model reaction involving the precursor of AMEF, 5-(azidomethyl)furfural (AMF) (3), following classical CuAAC methodology under mild conditions. To implement the bio-derived strategy, the available bulk constituents of natural biomass (cellulose and fructose) were tested as the feedstock for this synthesis.

The synthesis of compounds 3 and $\mathbf{6}$ was carried out using renewable resources as starting materials (Scheme 2). In the first stage, we conducted the Lewis or Brønsted acid catalyzed dehydration of natural carbohydrates (cellulose or fructose, respectively) into HMF (1), which was isolated in a pure crystalline form, as described previously. ${ }^{20}$

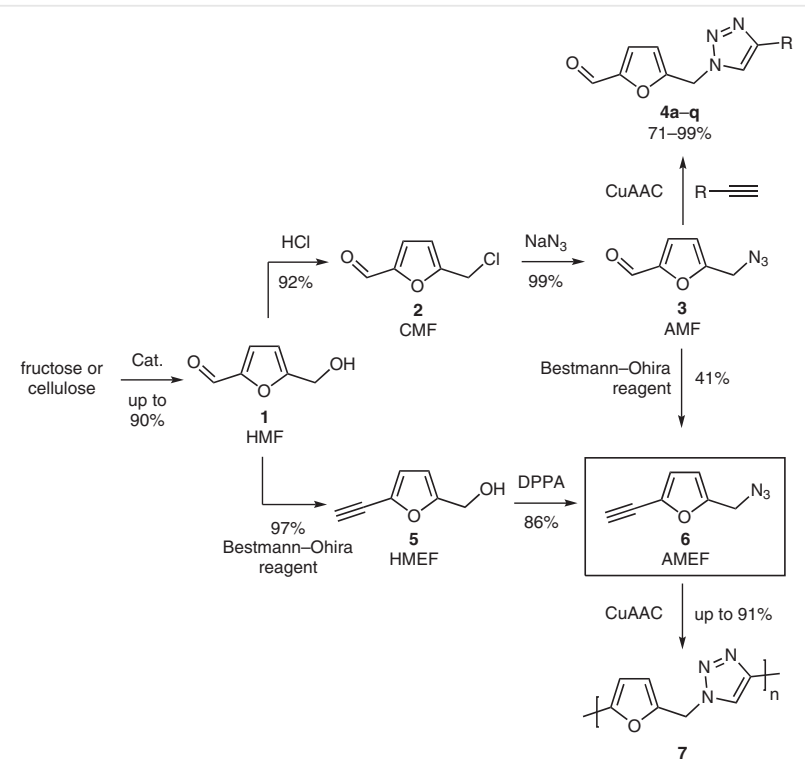

Scheme 2 A proposed synthetic strategy affording new bio-based building blocks and oligomers via click-chemistry approach
In the next stage, HMF was converted into 5-(chloromethyl)furfural (CMF) (2) using hydrogen chloride in a biphasic system, with the product being obtained in $92 \%$ yield. It should be noted that CMF itself can be discussed as a platform chemical since it can be obtained directly from biomass in good yield. ${ }^{21}$ Subsequent nucleophilic substitution of the chlorine in CMF with sodium azide under mild conditions afforded AMF (3) with almost complete conversion.

To probe the reactivity of the azide group linked to a furfural moiety, AMF was subsequently utilized in a series of CuAAC reactions with various alkynes. A range of new biheterocyclic compounds $\mathbf{4 a - q}$ was synthesized in good to excellent yields in these experiments (Scheme 3). All reactions were carried out under mild conditions in the presence of air, using aqueous ethanol as the solvent and with almost stoichiometric ratios of reagents without any purification of products. The use of classic CuAAC methodology is nicely compatible with a wide range of terminal alkynes in this cycloaddition reaction. Nevertheless, it is known that alkynes with internal triple bonds are significantly less reactive; for example, the reaction of AMF with deca-1,4diyne resulted in the formation of only one product $\mathbf{4 d}$ without involving the internal triple bond in the cycloaddition. In spite of the high practical interest of biomass conversion and furan-based platform chemicals, this work represents the first study of the reactivity of HMF-derived azide 3 in CuAAC reactions and the preparation of new compounds $\mathbf{4 a - q}{ }^{6}$

The synthesis of reactive self-clickable AMEF (6) was performed by two methods. The more efficient approach was a two-stage process starting with substitution of the aldehyde fragment in HMF with an acetylenic moiety using the Bestmann-Ohira reagent (dimethyl 1-diazo-2-oxopropylphosphonate). The formation of 2-hydroxymethyl-5ethynylfuran (HMEF) (5) in 97\% yield was followed by substitution of the hydroxy group with an azide moiety from diphenylphosphoryl azide (DPPA) in the presence of DBU. The yield after chromatography was $86 \%$; thus utilization of this methodology resulted in an overall yield of AMEF of 83\% from HMF.

Another method for the synthesis of AMEF involves the introduction of an acetylenic moiety on AMF via alkynylation of the aldehyde group by using potassium carbonate and the Bestmann-Ohira reagent. The target product 6 was isolated by column chromatography on silica gel in a moderate $41 \%$ yield (37\% overall yield from HMF) and showed relatively good stability at reduced temperatures (below $-5{ }^{\circ} \mathrm{C}$ ).

To evaluate the synthetic potential of AMEF as a monomer, we conducted a study of its 'click' polycondensation using different $\mathrm{Cu}$-based catalytic systems: aqueous ethanol as a green solvent with the classic copper(II) sulfate and sodium ascorbate couple, being an alternative to polycondensation in DMF with copper(I) bromide in the presence 

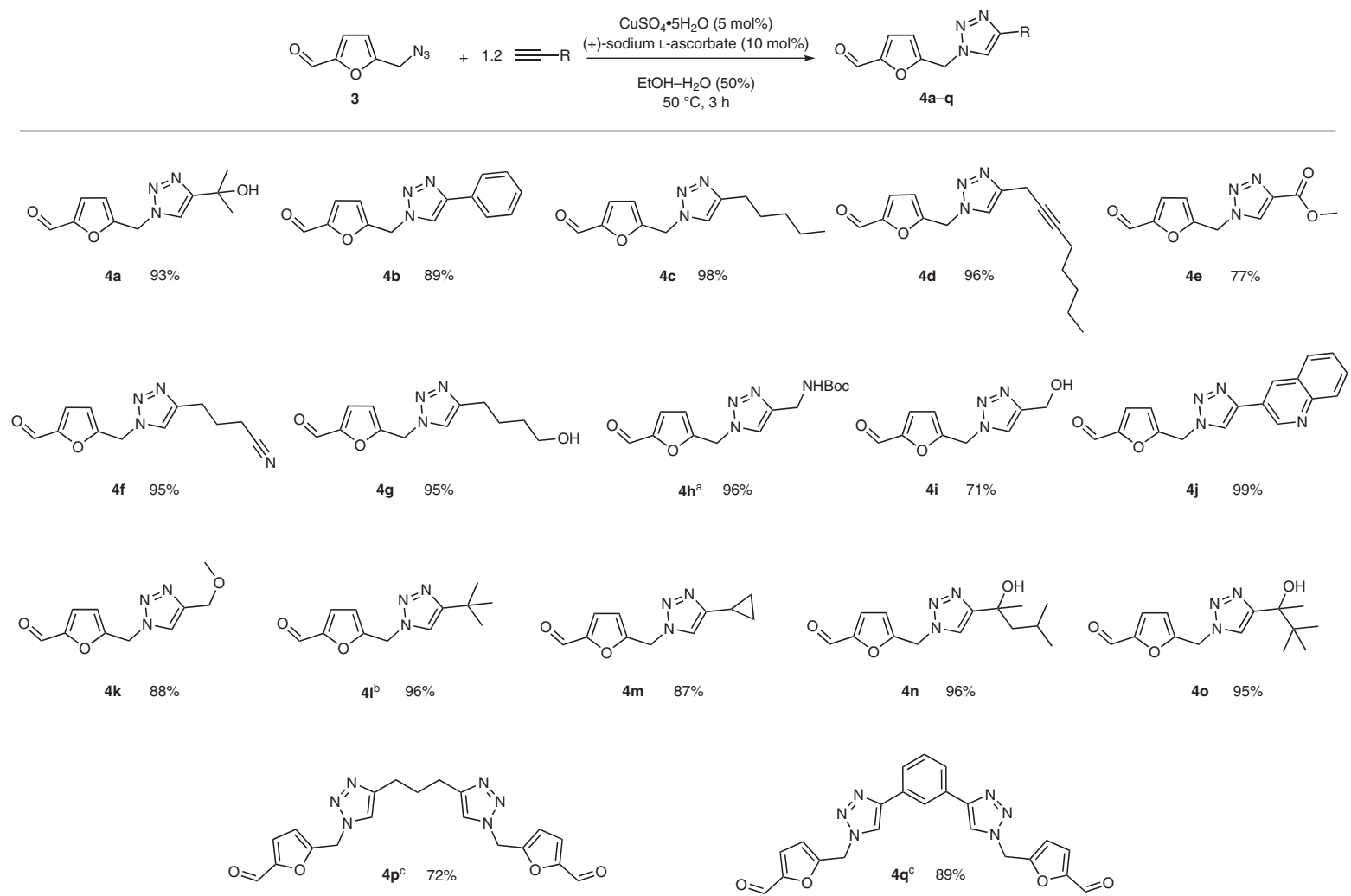

Scheme 3 Synthesis of triazole derivatives of AMF (isolated yields are shown); ${ }^{\text {a }} 1.05$ equiv of the corresponding alkyne was used; ${ }^{\text {b }} 1.5$ equiv of the corresponding alkyne was used; ${ }^{c} 0.45$ equiv of the corresponding alkyne was used

of different ligands (Table 1 ). The best performance was shown in the case of the CuBr/PMDETA system (Table 1, entry 1$)$.

Table 1 Cu-Catalyzed Polymerization of AMEF Using Different Catalytic Systems

\begin{tabular}{lllc}
\hline Entry & Catalyst & Solvent & Yield \\
\hline 1 & CuBr/PMDETA & DMF & $91 \%$ \\
2 & CuBr/TEEDA & DMF & $84 \%$ \\
3 & CuBr/bipy & DMF & $76 \%$ \\
4 & CuBr/phen & DMF & $79 \%$ \\
5 & CuSO $_{4} /$ sodium ascorbate & EtOH $/ \mathrm{H}_{2} \mathrm{O}$ & $70 \%$ \\
\hline
\end{tabular}

As a result of polycondensation, oligomers were obtained as colored solids (the color depended on the catalytic system used) that were slightly soluble in dipolar aprotic solvents such as DMF and DMSO. The number-average molecular weight of the oligomers was estimated roughly as $4100 \mathrm{~g} / \mathrm{mol}$ by integration of the terminal azide end-group signal (4.51 ppm) relative to the signals of the rest of the chain in the ${ }^{1} \mathrm{H}$ NMR spectra. The oligomers were found to be thermally transformable. This finding was confirmed by
DSC measurements (see the Supporting Information), as all of the oligomer samples showed strong exothermic effects at temperatures above $200^{\circ} \mathrm{C}$. Apparently, this is due to the presence of relatively energy-rich azide fragments in the material.

The microstructures of the prepared oligomer samples were studied by means of scanning electron microscopy (FE-SEM) (Figure 1). The substances had similar morphologies of sharp-edged particles whenever DMF was used as the solvent. Thus, the sample morphologies were almost independent of the used catalytic system and were tolerant to variations in the catalyst (Figure 1, A-D). To modify the morphology, aqueous ethanol was used as the reaction medium, leading to the formation of larger particles with smooth edges (Figure 1, E and F).

In conclusion, we have, for the first time, synthesized an ambivalent compound with both azide and alkyne moieties from renewable sources. The synthesis provides an access to a new type of biheterocyclic oligomers under mild conditions following classical click-chemistry methodology. The oligomers were characterized by NMR, IR, DSC and FE-SEM studies. In addition, a broad range of triazoles with a furfural moiety was prepared in good to excellent yields in accordance with green chemistry requirements. The approach paves the way for the application of Cu-catalyzed 

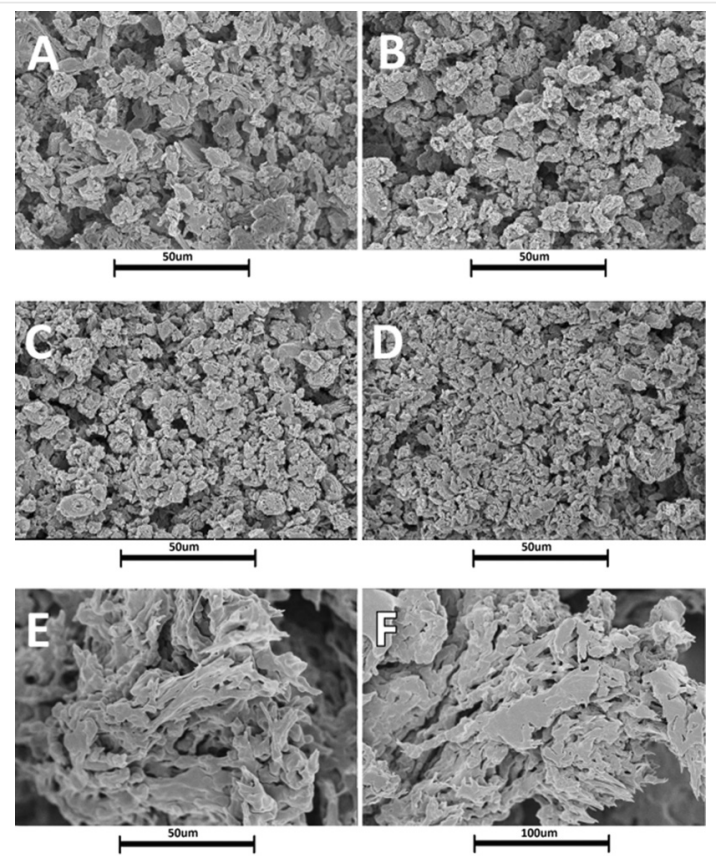

Figure 1 Field-emission scanning electron microscopy (FE-SEM) images of the synthesized oligomers prepared from $\operatorname{AMEF}(\mathbf{6})$ using the catalytic systems listed in Table 1 (entries 1-5): 1 (A), 2 (B), 3 (C), 4 (D) and 5 (E and $F) ; 50 \mu \mathrm{m}$ scale bars are shown below images $A-E$ and a 100 $\mu \mathrm{m}$ scale bar is shown below image $F$.

systems for the construction of bio-based biheterocyclic compounds and is expected to provide attractive solutions for the development of new building blocks and the design of bio-based organic materials. The direct involvement of natural biomass as a sustainable source of chemicals is one of the most promising trends in synthetic chemistry and more detailed studies on the subject are to be anticipated in the near future.

Caution! Sodium azide is highly toxic and should be handled with extreme care. All reagents from commercial sources were checked by NMR before use or were purified by standard methods. ${ }^{22}$ HMF (1), ${ }^{20}$ CMF (2), ${ }^{\text {5e }} \mathrm{N}$-Boc-propargylamine, ${ }^{23} \operatorname{HMEF}(\mathbf{5}),{ }^{12}$ diphenylphosphoryl azide (DPPA) ${ }^{24}$ and dimethyl 1-diazo-2-oxopropylphosphonate (Bestmann-Ohira reagent $)^{25}$ were prepared according to the published procedures. All reactions were performed in oven-dried $\left(120^{\circ} \mathrm{C}\right)$ glassware. Chromatographic separations were performed on silica gel (Kieselgel, 230-400 mesh, Merck Schuchardt) with analytical grade solvents. Analytical TLC was performed on Merck silica gel plates with QF-254 indicator. Visualization was accomplished with UV light (254 $\mathrm{nm})$. Melting points were determined using a 1101D Mel-Temp apparatus and are uncorrected. IR spectra were recorded on a Bruker Alpha-T spectrophotometer. NMR spectra were recorded using a Bruker Fourier $300 \mathrm{HD}$ spectrometer at frequencies of $300.1 \mathrm{MHz}\left({ }^{1} \mathrm{H}\right)$ and $75.5 \mathrm{MHz}\left({ }^{13} \mathrm{C}\right)$ with the residual solvent peak as an internal standard. Unless otherwise noted, NMR spectra were recorded at room temperature. The spectra were processed using MestReNova software. The ${ }^{13} \mathrm{C}$ NMR spectra of compounds $4 \mathbf{a}, 4 \mathbf{c}, \mathbf{4 f}, \mathbf{4 i}, \mathbf{4 k}, \mathbf{4 m}$ and $\mathbf{4 n}$ were also recorded at $-60{ }^{\circ} \mathrm{C}$ (Bruker Avance 600 NMR spectrometer, 151 $\mathrm{MHz}$ ), since at room temperature two of the signals were rather broad and difficult to detect, most likely due to dynamic or relaxation properties (see the Supporting Information). Electrospray ionization mass spectrometry (ESI-MS) was performed on a high-resolution time-offlight Bruker maXis instrument. The spectra were processed using the Bruker Data Analysis 4.0 software package. Analysis of sample morphology was carried out using a Hitachi SU8000 field-emission scanning electron microscope. DSC analysis was performed on a Mettler Toledo DSC823e calorimeter equipped with an FSR 5 thermocouple and a liquid nitrogen cooling block. The samples were placed in $40 \mu \mathrm{L}$ aluminum crucibles with perforated caps. Perforation allowed free atmosphere exchange with the oven to obtain results at a constant pressure. The samples were heated under an argon atmosphere (flow: $70 \mathrm{~mL} / \mathrm{min}$ ). Data processing was performed using the STARe service program.

\section{5-(Azidomethyl)furfural (AMF) (3)}

Sodium azide ( $4.5 \mathrm{~g}, 69.2 \mathrm{mmol}$ ) was added to a solution of 5-(chloromethyl)furfural $(5.0 \mathrm{~g}, 34.6 \mathrm{mmol})$ in acetonitrile $(100 \mathrm{~mL})$. The mixture was stirred for $6 \mathrm{~h}$ at room temperature, filtered through Celite, and the filtrate was evaporated at $30^{\circ} \mathrm{C}$. The residue was dissolved in diethyl ether and refiltered through Celite to remove inorganic impurities. After evaporation, 5-(azidomethyl)furfural (3) was obtained as a yellow oil $(5.18 \mathrm{~g}, 99 \%)$.

IR (KBr): 3123, 2838, 2101, 1678, 1522, 1402, 1275, 1024, $810 \mathrm{~cm}^{-1}$.

${ }^{1} \mathrm{H} \mathrm{NMR}\left(300 \mathrm{MHz}, \mathrm{CDCl}_{3}\right): \delta=4.40(\mathrm{~s}, 2 \mathrm{H}), 6.54(\mathrm{~d}, J=3.5 \mathrm{~Hz}, 1 \mathrm{H})$, $7.21(\mathrm{~d}, J=3.5 \mathrm{~Hz}, 1 \mathrm{H}), 9.62(\mathrm{~s}, 1 \mathrm{H})$.

${ }^{13} \mathrm{C}$ NMR $\left(75 \mathrm{MHz}, \mathrm{CDCl}_{3}\right): \delta=47.1,111.5,121.9,153.1,155.4,177.7$. HRMS (ESI): $m / z[\mathrm{M}+\mathrm{Na}]^{+}$calcd for $\mathrm{C}_{6} \mathrm{H}_{5} \mathrm{~N}_{3} \mathrm{O}_{2} \mathrm{Na}$ : 174.0274; found: 174.0279.

\section{Dipolar Cycloaddition of 5-(Azidomethyl)furfural (3) to Alkynes; General Procedure}

5-(Azidomethyl)furfural (3) (200 mg, $1.32 \mathrm{mmol}$ ) in an $8 \mathrm{~mL}$ glass vial was dissolved in $50 \%$ aqueous ethanol $(3 \mathrm{~mL}$ ) followed by the addition of copper sulfate pentahydrate ( $16 \mathrm{mg}, 0.07 \mathrm{mmol}$ ) and sodium ascorbate (26 mg, $0.13 \mathrm{mmol}$ ). Next, an appropriate amount of alkyne (typically $1.58 \mathrm{mmol} ; 1.38 \mathrm{mmol}$ of $N$-Boc-propargylamine; $1.98 \mathrm{mmol}$ of 3,3-dimethylbut-1-yne) was added, and the reaction mixture was stirred at $50{ }^{\circ} \mathrm{C}$ for $3 \mathrm{~h}$. For triazoles insoluble in aqueous ethanol $(\mathbf{4 b}$, $\mathbf{4 e}, \mathbf{4 j})$, the reaction mixture was diluted with water $(10 \mathrm{~mL})$ and the precipitate was filtered, washed with water and dried under vacuum. Drying was completed in a desiccator over phosphorus pentoxide. For triazoles soluble in aqueous ethanol $(\mathbf{4 a}, \mathbf{c}, \mathbf{d}, \mathbf{f}-\mathbf{i}, \mathbf{k}-\mathbf{o})$, the solvent was evaporated under vacuum, the residue dissolved in chloroform and filtered through Celite. Subsequent evaporation under vacuum at $50{ }^{\circ} \mathrm{C}$ yielded the pure triazoles.

\section{5-\{[4-(2-hydroxypropan-2-yl)-1H-1,2,3-triazol-1-yl]methyl\}furfu-} ral (4a)

Yield: $288 \mathrm{mg}$ (93\%); yellowish-brown crystals; $\mathrm{mp} 85-88^{\circ} \mathrm{C}$.

IR (KBr): 3322, 3139, 1668, 1527, 1403, 1019, 977, $792 \mathrm{~cm}^{-1}$.

${ }^{1} \mathrm{H}$ NMR $\left(300 \mathrm{MHz}, \mathrm{CD}_{2} \mathrm{Cl}_{2}\right): \delta=1.58(\mathrm{~s}, 6 \mathrm{H}), 5.62(\mathrm{~s}, 2 \mathrm{H}), 6.63(\mathrm{~d}, J=$ $3.5 \mathrm{~Hz}, 1 \mathrm{H}$ ), $7.22(\mathrm{~d}, J=3.5 \mathrm{~Hz}, 1 \mathrm{H}), 7.68$ (br s, $1 \mathrm{H}), 9.58(\mathrm{~s}, 1 \mathrm{H})$.

${ }^{13} \mathrm{C}$ NMR $\left(151 \mathrm{MHz}, \mathrm{CDCl}_{3},-60{ }^{\circ} \mathrm{C}\right): \delta=29.9,46.3,67.8,112.9,120.0$, 125.6, 152.1, 153.6, 156.1, 177.8 .

HRMS (ESI): $m / z[\mathrm{M}+\mathrm{Na}]^{+}$calcd for $\mathrm{C}_{11} \mathrm{H}_{13} \mathrm{~N}_{3} \mathrm{O}_{3} \mathrm{Na}$ : 258.0849; found: 258.0845 . 
5-[(4-Phenyl-1H-1,2,3-triazol-1-yl)methyl]furfural (4b)

Yield: $298 \mathrm{mg}$ (89\%); yellow crystals; $\mathrm{mp} 122-125^{\circ} \mathrm{C}$. IR (KBr): 3116, 1676, 1525, 1195, 1027, 765, $693 \mathrm{~cm}^{-1}$. ${ }^{1} \mathrm{H} \mathrm{NMR}\left(300 \mathrm{MHz}, \mathrm{CDCl}_{3}\right): \delta=5.68(\mathrm{~s}, 2 \mathrm{H}), 6.63(\mathrm{~d}, J=3.6 \mathrm{~Hz}, 1 \mathrm{H})$, 7.23 (d, $J=3.6 \mathrm{~Hz}, 1 \mathrm{H}), 7.32-7.37$ ( $\mathrm{m}, 1 \mathrm{H}), 7.41-7.46$ (m, $2 \mathrm{H}), 7.81-$ $7.84(\mathrm{~m}, 2 \mathrm{H}), 7.90(\mathrm{~s}, 1 \mathrm{H}), 9.65(\mathrm{~s}, 1 \mathrm{H})$.

${ }^{13} \mathrm{C}$ NMR $\left(75 \mathrm{MHz}, \mathrm{CDCl}_{3}\right): \delta=46.9,112.5,120.0,122.1,126.0,128.6$, $129.1,130.2,148.7,153.3,153.5,177.7$.

HRMS (ESI): $m / z[M+H]^{+}$calcd for $\mathrm{C}_{14} \mathrm{H}_{12} \mathrm{~N}_{3} \mathrm{O}_{2}$ : 254.0924; found: 254.0930 .

\section{5-[(4-Pentyl-1H-1,2,3-triazol-1-yl)methyl]furfural (4c)}

Yield: $320 \mathrm{mg}$ (98\%); yellowish-green crystals; mp 63-66 ${ }^{\circ} \mathrm{C}$.

IR (KBr): 3065, 2927, 1674, 1532, 1266, 1018, 977, $799 \mathrm{~cm}^{-1}$.

${ }^{1} \mathrm{H} \mathrm{NMR}\left(300 \mathrm{MHz}, \mathrm{CDCl}_{3}\right): \delta=0.88(\mathrm{t}, J=6.6 \mathrm{~Hz}, 3 \mathrm{H}), 1.32-1.34(\mathrm{~m}, 4$ H), 1.67-1.71 (m, $2 \mathrm{H}), 2.70(\mathrm{br} \mathrm{s}, 2 \mathrm{H}), 5.60(\mathrm{~s}, 2 \mathrm{H}), 6.56(\mathrm{~d}, J=3.5 \mathrm{~Hz}$, $1 \mathrm{H}), 7.20$ (d, J = $3.5 \mathrm{~Hz}, 1 \mathrm{H}), 7.49$ (br s, $1 \mathrm{H}), 9.62(\mathrm{~s}, 1 \mathrm{H})$.

${ }^{13} \mathrm{C}$ NMR (151 MHz, $\left.\mathrm{CDCl}_{3},-60{ }^{\circ} \mathrm{C}\right): \delta=14.3,22.5,25.5,29.1,31.4$, $46.3,112.5,121.3,125.5,149.2,152.0,153.9,177.6$.

HRMS (ESI): $m / z[M+H]^{+}$calcd for $\mathrm{C}_{13} \mathrm{H}_{18} \mathrm{~N}_{3} \mathrm{O}_{2}$ : 248.1394; found: 248.1392 .

\section{5-\{[4-(Oct-2-yn-1-yl)-1H-1,2,3-triazol-1-yl]methyl\}furfural (4d)} Yield: $362 \mathrm{mg}$ (96\%); brown oil.

IR (KBr): 3128, 2930, 1681, 1523, 1403, 1024, $790 \mathrm{~cm}^{-1}$.

${ }^{1} \mathrm{H} \mathrm{NMR}\left(300 \mathrm{MHz}, \mathrm{CDCl}_{3}\right): \delta=0.88(\mathrm{t}, J=7.0 \mathrm{~Hz}, 3 \mathrm{H}), 1.29-1.36(\mathrm{~m}, 4$ H), 1.45-1.54 (m, $2 \mathrm{H}), 2.14-2.20(\mathrm{~m}, 2 \mathrm{H}), 3.67(\mathrm{~s}, 2 \mathrm{H}), 5.60(\mathrm{~s}, 2 \mathrm{H})$, $6.58(\mathrm{~d}, J=3.5 \mathrm{~Hz}, 1 \mathrm{H}), 7.20(\mathrm{~d}, J=3.5 \mathrm{~Hz}, 1 \mathrm{H}), 7.60(\mathrm{~s}, 1 \mathrm{H}), 9.62(\mathrm{~s}, 1$ $\mathrm{H})$.

${ }^{13} \mathrm{C}$ NMR $\left(75 \mathrm{MHz}, \mathrm{CDCl}_{3}\right): \delta=14.1,16.8,18.9,22.3,28.6,31.2,46.8$, 75.6, 82.7, 112.4, 122.0, 146.1, 153.2, 153.6, 177.7.

HRMS (ESI): $m / z[M+H]^{+}$calcd for $\mathrm{C}_{16} \mathrm{H}_{20} \mathrm{~N}_{3} \mathrm{O}_{2}$ : 286.1550; found: 286.1551 .

\section{5-[(4-Methoxycarbonyl-1H-1,2,3-triazol-1-yl)methyl]furfural (4e)} Yield: $240 \mathrm{mg}$ (77\%); red crystals; mp $125-127^{\circ} \mathrm{C}$.

IR (KBr): 3123, 2838, 1727, 1674, 1548, 1333, 1241, 1017, $813 \mathrm{~cm}^{-1}$. ${ }^{1} \mathrm{H}$ NMR (300 MHz, DMSO- $d_{6}$ ): $\delta=3.84(\mathrm{~s}, 3 \mathrm{H}$ ), 5.88 (s, $2 \mathrm{H}$ ), 6.83 (d, $J=3.6 \mathrm{~Hz}, 1 \mathrm{H}), 7.53(\mathrm{~d}, J=3.6 \mathrm{~Hz}, 1 \mathrm{H}), 8.88(\mathrm{~s}, 1 \mathrm{H}), 9.57(\mathrm{~s}, 1 \mathrm{H})$.

${ }^{13} \mathrm{C}$ NMR $\left(75 \mathrm{MHz}\right.$, DMSO- $d_{6}$ ): $\delta=46.1,51.8,112.6,123.9,129.5$, $138.8,152.5,153.9,160.5,178.4$.

HRMS (ESI): $m / z[\mathrm{M}+\mathrm{H}]^{+}$calcd for $\mathrm{C}_{10} \mathrm{H}_{10} \mathrm{~N}_{3} \mathrm{O}_{4}$ : 236.0666; found: 236.0667.

\section{5-\{[4-(3-Cyanopropyl)-1H-1,2,3-triazol-1-yl]methyl\}furfural (4f)}

Yield: $306 \mathrm{mg}$ (95\%); brown crystals; $\mathrm{mp} 70-74^{\circ} \mathrm{C}$.

IR (KBr): 3113, 2243, 1675, 1530, 1270, 972, $795 \mathrm{~cm}^{-1}$.

${ }^{1} \mathrm{H} \mathrm{NMR}\left(300 \mathrm{MHz}, \mathrm{CDCl}_{3}\right.$ ): $\delta=2.06$ (br s, $2 \mathrm{H}$ ), 2.40-2.44 (m, $2 \mathrm{H}$ ), 2.86 (br s, $2 \mathrm{H}), 5.61(\mathrm{~s}, 2 \mathrm{H}), 6.58(\mathrm{~d}, J=3.5 \mathrm{~Hz}, 1 \mathrm{H}), 7.20(\mathrm{~d}, J=3.5 \mathrm{~Hz}$, $1 \mathrm{H}), 7.61$ (br s, $1 \mathrm{H}), 9.59$ (s, $1 \mathrm{H}$ ).

${ }^{13} \mathrm{C}$ NMR $\left(151 \mathrm{MHz}, \mathrm{CDCl}_{3},-60{ }^{\circ} \mathrm{C}\right): \delta=16.6,24.0,24.5,46.4,112.7$, $119.9,122.0,125.5,146.2,152.1,153.5,177.7$.

HRMS (ESI): $m / z[M+H]^{+}$calcd for $\mathrm{C}_{12} \mathrm{H}_{13} \mathrm{~N}_{4} \mathrm{O}_{2}$ : 245.1033; found: 245.1037.
5-\{[4-(4-Hydroxybutyl)-1H-1,2,3-triazol-1-yl]methyl\}furfural (4g) Yield: $314 \mathrm{mg}$ (95\%); yellow crystals; $\mathrm{mp} 60-63{ }^{\circ} \mathrm{C}$.

IR (KBr): 3344, 3118, 2934, 1681, 1525, 1264, 1027, $774 \mathrm{~cm}^{-1}$.

${ }^{1} \mathrm{H}$ NMR $\left(300 \mathrm{MHz}, \mathrm{CDCl}_{3}\right): \delta=1.57-1.66(\mathrm{~m}, 2 \mathrm{H}), 1.70-1.80(\mathrm{~m}, 2 \mathrm{H})$, 1.95 (br s, $1 \mathrm{H}), 2.74(\mathrm{t}, J=7.4 \mathrm{~Hz}, 2 \mathrm{H}), 3.65(\mathrm{t}, J=6.3 \mathrm{~Hz}, 2 \mathrm{H}), 5.58(\mathrm{~s}$, $2 \mathrm{H}), 6.55(\mathrm{~d}, J=3.6 \mathrm{~Hz}, 1 \mathrm{H}), 7.20(\mathrm{~d}, J=3.6 \mathrm{~Hz}, 1 \mathrm{H}), 7.44(\mathrm{~s}, 1 \mathrm{H}), 9.60$ ( $\mathrm{s}, 1 \mathrm{H})$.

${ }^{13} \mathrm{C}$ NMR $\left(75 \mathrm{MHz}, \mathrm{CDCl}_{3}\right): \delta=25.4,25.6,32.2,46.7,62.4,112.3,121.2$, 122.2, 148.9, 153.1, 153.8, 177.7.

HRMS (ESI): $m / z[M+H]^{+}$calcd for $\mathrm{C}_{12} \mathrm{H}_{16} \mathrm{~N}_{3} \mathrm{O}_{3}: 250.1186$; found: 250.1186 .

5-\{[4-(N-Boc-aminomethyl)-1H-1,2,3-triazol-1-yl]methyl\}furfural (4h)

Yield: $388 \mathrm{mg}$ (96\%); yellow crystals; $\mathrm{mp} 106-110^{\circ} \mathrm{C}$.

IR (KBr): 3403, 1683, 1512, 1272, 1169, $793 \mathrm{~cm}^{-1}$.

${ }^{1} \mathrm{H}$ NMR $\left(300 \mathrm{MHz}, \mathrm{CDCl}_{3}\right): \delta=1.41(\mathrm{~s}, 9 \mathrm{H}), 4.38(\mathrm{~d}, J=5.9 \mathrm{~Hz}, 2 \mathrm{H})$, 5.15 (br s, $1 \mathrm{H}), 5.59(\mathrm{~s}, 2 \mathrm{H}), 6.56(\mathrm{~d}, J=3.2 \mathrm{~Hz}, 1 \mathrm{H}), 7.20(\mathrm{~d}, J=3.4 \mathrm{~Hz}$, $1 \mathrm{H}), 7.63(\mathrm{~s}, 1 \mathrm{H}), 9.62(\mathrm{~s}, 1 \mathrm{H})$.

${ }^{13} \mathrm{C}$ NMR $\left(75 \mathrm{MHz}, \mathrm{CDCl}_{3}\right): \delta=28.5,36.2,46.8,79.9,112.5,121.8$, $122.3,146.5,153.3,153.4,155.9,177.7$.

HRMS (ESI): $m / z[M+H]^{+}$calcd for $\mathrm{C}_{14} \mathrm{H}_{19} \mathrm{~N}_{4} \mathrm{O}_{4}: 307.1401$; found: 307.1401.

\section{5-[(4-Hydroxymethyl-1H-1,2,3-triazol-1-yl)methyl]furfural (4i)}

Yield: $195 \mathrm{mg}$ (71\%); yellow crystals; $\mathrm{mp} 75-79^{\circ} \mathrm{C}$.

IR (KBr): 3370, 1662, 1528, 1267, 1133, 1013, $795 \mathrm{~cm}^{-1}$.

$\left.{ }^{1} \mathrm{H} \mathrm{NMR} \mathrm{(300} \mathrm{MHz,} \mathrm{CDCl}_{3}\right): \delta=2.59$ (br s, $\left.1 \mathrm{H}\right), 4.79$ (br s, $\left.2 \mathrm{H}\right), 5.61$ (s, $2 \mathrm{H}), 6.59$ (d, J = 3.5 Hz, $1 \mathrm{H}), 7.20(\mathrm{~d}, J=3.5 \mathrm{~Hz}, 1 \mathrm{H}), 7.77$ (br s, $1 \mathrm{H}$ ), $9.60(\mathrm{~s}, 1 \mathrm{H})$.

${ }^{13} \mathrm{C}$ NMR $\left(151 \mathrm{MHz}, \mathrm{CDCl}_{3},-60{ }^{\circ} \mathrm{C}\right): \delta=46.5,54.9,112.9,122.9,125.5$, 148.2, 152.1, 153.4, 177.7.

HRMS (ESI): $m / z[M+H]^{+}$calcd for $\mathrm{C}_{9} \mathrm{H}_{10} \mathrm{~N}_{3} \mathrm{O}_{3}$ : 208.0717; found: 208.0715 .

5-\{[4-(Quinolin-3-yl)-1H-1,2,3-triazol-1-yl]methyl\}furfural (4j) Yield: $398 \mathrm{mg}$ (99\%); orange crystals; mp $179-183^{\circ} \mathrm{C}$. IR (KBr): 3424, 3130, 1680, 1497, 1030, 827, 786, $759 \mathrm{~cm}^{-1}$.

${ }^{1} \mathrm{H}$ NMR $\left(300 \mathrm{MHz}\right.$, DMSO- $\left.d_{6}\right): \delta=5.94(\mathrm{~s}, 2 \mathrm{H}), 6.90(\mathrm{~d}, J=3.5 \mathrm{~Hz}, 1$ $\mathrm{H}), 7.57(\mathrm{~d}, J=3.5 \mathrm{~Hz}, 1 \mathrm{H}), 7.61-7.66(\mathrm{~m}, 1 \mathrm{H}), 7.77(\mathrm{t}, J=7.3 \mathrm{~Hz}, 1 \mathrm{H})$, $8.06(\mathrm{~d}, J=8.0 \mathrm{~Hz}, 2 \mathrm{H}), 8.82$ (s, $1 \mathrm{H}), 8.91$ (s, $1 \mathrm{H}), 9.44$ (br s, $1 \mathrm{H}), 9.59$ (s, $1 \mathrm{H})$.

${ }^{13} \mathrm{C}$ NMR (151 MHz, DMSO- $d_{6}$ ): $\delta=46.2,112.7,122.7,123.7,124.0$, 127.2, 127.6, 128.3, 128.8, 129.7, 131.1, 144.2, 147.1, 148.2, 152.6, $154.3,178.5$.

HRMS (ESI): $m / z[M+H]^{+}$calcd for $\mathrm{C}_{17} \mathrm{H}_{13} \mathrm{~N}_{4} \mathrm{O}_{2}: 305.1033$; found: 305.1032 .

\section{5-[(4-Methoxymethyl-1 H-1,2,3-triazol-1-yl)methyl]furfural (4k)} Yield: $257 \mathrm{mg}$ (88\%); brown oil.

IR (KBr): 3126, 2932, 1681, 1524, 1402, 1274, 1195, 1094, $793 \mathrm{~cm}^{-1}$. ${ }^{1} \mathrm{H} \mathrm{NMR}\left(300 \mathrm{MHz}, \mathrm{CDCl}_{3}\right): \delta=3.40(\mathrm{~s}, 3 \mathrm{H}), 4.54(\mathrm{~s}, 2 \mathrm{H}), 5.61(\mathrm{~s}, 2 \mathrm{H})$, $6.56(\mathrm{~d}, J=3.5 \mathrm{~Hz}, 1 \mathrm{H}), 7.19(\mathrm{~d}, J=3.5 \mathrm{~Hz}, 1 \mathrm{H}), 7.78(\mathrm{br} \mathrm{s}, 1 \mathrm{H}), 9.60(\mathrm{~s}$, $1 \mathrm{H})$. 
${ }^{13} \mathrm{C}$ NMR $\left(151 \mathrm{MHz}, \mathrm{CDCl}_{3},-60{ }^{\circ} \mathrm{C}\right): \delta=29.9,46.3,67.8,112.9,120.0$, 125.6, 152.1, 153.6, 156.1, 177.8 .

HRMS (ESI): $m / z[M+H]^{+}$calcd for $\mathrm{C}_{10} \mathrm{H}_{12} \mathrm{~N}_{3} \mathrm{O}_{3}$ : 222.0873; found: 222.0876 .

\section{5-\{[4-(tert-Butyl)-1H-1,2,3-triazol-1-yl]methyl\}furfural (4l)}

Yield: $298 \mathrm{mg}$ (96\%); yellow crystals; $\mathrm{mp} 98-101{ }^{\circ} \mathrm{C}$.

IR (KBr): 3123, 2966, 1674, 1526, 1221, 1050, $763 \mathrm{~cm}^{-1}$.

${ }^{1} \mathrm{H}$ NMR (300 MHz, $\mathrm{CDCl}_{3}$ ): $\delta=1.33(\mathrm{~s}, 9 \mathrm{H}), 5.57$ (s, $2 \mathrm{H}$ ), 6.55 (d, $J=$ $3.5 \mathrm{~Hz}, 1 \mathrm{H}), 7.20$ (d, J = 3.5 Hz, $1 \mathrm{H}), 7.36(\mathrm{~s}, 1 \mathrm{H}), 9.62(\mathrm{~s}, 1 \mathrm{H})$.

${ }^{13} \mathrm{C}$ NMR (75 MHz, $\mathrm{CDCl}_{3}$ ): $\delta=30.4,30.9,46.6,112.3,118.9,122.2$, 153.1, 154.0, 158.6, 177.7 .

HRMS (ESI): $m / z[M+H]^{+}$calcd for $\mathrm{C}_{12} \mathrm{H}_{16} \mathrm{~N}_{3} \mathrm{O}_{2}$ : 234.1237; found: 234.1235 .

\section{5-[(4-Cyclopropyl-1H-1,2,3-triazol-1-yl)methyl]furfural (4m)}

Yield: $250 \mathrm{mg}$ (87\%); yellow crystals; $\mathrm{mp} 99-102{ }^{\circ} \mathrm{C}$.

IR (KBr): 3088, 1673, 1525, 1016, 976, $765 \mathrm{~cm}^{-1}$.

${ }^{1} \mathrm{H} \mathrm{NMR}\left(300 \mathrm{MHz}, \mathrm{CDCl}_{3}\right): \delta=0.83-0.94(\mathrm{~m}, 4 \mathrm{H}), 1.93$ (br s, $1 \mathrm{H}$ ), $5.55(\mathrm{~s}, 2 \mathrm{H}), 6.54(\mathrm{~d}, J=3.5 \mathrm{~Hz}, 1 \mathrm{H}), 7.19(\mathrm{~d}, J=3.5 \mathrm{~Hz}, 1 \mathrm{H}), 7.45$ (br s, $1 \mathrm{H}), 9.60(\mathrm{~s}, 1 \mathrm{H})$.

${ }^{13} \mathrm{C}$ NMR $\left(151 \mathrm{MHz}, \mathrm{CDCl}_{3},-60{ }^{\circ} \mathrm{C}\right): \delta=6.7,8.3,46.3,112.5,120.3$, 125.6, 151.0, 152.0, 153.9, 177.7 .

HRMS (ESI): $m / z[M+H]^{+}$calcd for $\mathrm{C}_{11} \mathrm{H}_{12} \mathrm{~N}_{3} \mathrm{O}_{2}$ : 218.0924; found: 218.0927.

\section{5-\{[4-(2-Hydroxy-4-methylpentan-2-yl)-1H-1,2,3-triazol-1-} yl]methyl\}furfural (4n)

Yield: $352 \mathrm{mg}$ (96\%); brown oil.

IR (KBr): 3409, 2956, 1681, 1523, 1195, 1049, $789 \mathrm{~cm}^{-1}$.

${ }^{1} \mathrm{H} \mathrm{NMR}\left(300 \mathrm{MHz}, \mathrm{CDCl}_{3}\right): \delta=0.80(\mathrm{~d}, J=5.5 \mathrm{~Hz}, 3 \mathrm{H}), 0.85(\mathrm{~d}, J=5.5$ $\mathrm{Hz}, 3 \mathrm{H}), 1.58$ (s, $3 \mathrm{H}), 1.68-1.80(\mathrm{~m}, 3 \mathrm{H}), 2.61$ (br s, $1 \mathrm{H}), 5.60(\mathrm{~s}, 2 \mathrm{H})$, $6.55(\mathrm{~d}, J=1.6 \mathrm{~Hz}, 1 \mathrm{H}), 7.20$ (d, J = 2.1 Hz, $1 \mathrm{H}$ ), 7.60 (br s, $1 \mathrm{H}$ ), 9.60 (s, $1 \mathrm{H})$.

${ }^{13} \mathrm{C}$ NMR (151 MHz, $\left.\mathrm{CDCl}_{3},-60{ }^{\circ} \mathrm{C}\right): \delta=24.28,24.29,24.34,28.4,46.4$, 50.8, 71.1, 112.7, 120.4, 125.6, 152.1, 153.7, 155.9, 177.7 .

HRMS (ESI): $m / z[\mathrm{M}+\mathrm{H}]^{+}$calcd for $\mathrm{C}_{14} \mathrm{H}_{20} \mathrm{~N}_{3} \mathrm{O}_{3}$ : 278.1499; found: 278.1501 .

\section{5-\{[4-(2-Hydroxy-3,3-dimethylbutan-2-yl)-1H-1,2,3-triazol-1-} yl]methyl\}furfural (4o)

Yield: $349 \mathrm{mg}$ (95\%); brown crystals; $\mathrm{mp} 96-99^{\circ} \mathrm{C}$.

IR (KBr): 3426, 2965, 1681, 1523, 1376, 1210, 1071, $774 \mathrm{~cm}^{-1}$.

${ }^{1} \mathrm{H}$ NMR (300 MHz, DMSO- $d_{6}$ ): $\delta=0.85(\mathrm{~s}, 9 \mathrm{H}), 1.44(\mathrm{~s}, 3 \mathrm{H}), 4.88(\mathrm{br}$ s, $1 \mathrm{H}), 5.76(\mathrm{~s}, 2 \mathrm{H}), 6.74(\mathrm{~d}, J=3.5 \mathrm{~Hz}, 1 \mathrm{H}), 7.52(\mathrm{~d}, J=3.5 \mathrm{~Hz}, 1 \mathrm{H})$, $7.89(\mathrm{~s}, 1 \mathrm{H}), 9.56(\mathrm{~s}, 1 \mathrm{H})$.

${ }^{13} \mathrm{C}$ NMR $\left(75 \mathrm{MHz}\right.$, DMSO- $\left.d_{6}\right): \delta=23.6,25.3,37.3,45.6,74.0,112.1$, 123.0, 124.1, 152.3, 154.4, 155.0, 178.3.

HRMS (ESI): $m / z[M+H]^{+}$calcd for $\mathrm{C}_{14} \mathrm{H}_{20} \mathrm{~N}_{3} \mathrm{O}_{3}$ : 278.1499; found: 278.1495 .

\section{Dipolar Cycloaddition of 5-(Azidomethyl)furfural (3) to Com- pounds with Two Terminal Alkyne Moieties}

5-(Azidomethyl)furfural (3) (200 mg, $1.32 \mathrm{mmol}$ ) in an $8 \mathrm{~mL}$ glass vial was dissolved in $50 \%$ aqueous ethanol $(3 \mathrm{~mL}$ ) and then copper sulfate pentahydrate (16 mg, $0.07 \mathrm{mmol}$ ) and sodium ascorbate ( $26 \mathrm{mg}, 0.13$ mmol) were added. Next, the corresponding alkyne ( $0.60 \mathrm{mmol})$ was added and the reaction mixture was stirred at $50^{\circ} \mathrm{C}$ for $3 \mathrm{~h}$. The mixture was diluted with water $(10 \mathrm{~mL})$ and the precipitate was filtered, washed with water and dried under vacuum. Drying was completed in a desiccator over phosphorus pentoxide.

5,5'-\{[Propane-1,3-diylbis(1H-1,2,3-triazole-4,1-diyl)]bis(methylene)\}bis(furfural) (4p)

Yield: $171 \mathrm{mg}$ (72\%); white crystals; $\mathrm{mp} 141-144^{\circ} \mathrm{C}$.

IR (KBr): 3063, 1673, 1532, 1221, 1018, $797 \mathrm{~cm}^{-1}$.

${ }^{1} \mathrm{H}$ NMR (300 MHz, DMSO- $d_{6}$ ): $\delta=1.91$ (quin, $J=7.7 \mathrm{~Hz}, 2 \mathrm{H}$ ), 2.66 (t, $J=7.6 \mathrm{~Hz}, 4 \mathrm{H}), 5.75(\mathrm{~s}, 4 \mathrm{H}), 6.76(\mathrm{~d}, J=3.6 \mathrm{~Hz}, 2 \mathrm{H}), 7.51(\mathrm{~d}, J=3.6 \mathrm{~Hz}$, $2 \mathrm{H}), 7.95$ (s, $2 \mathrm{H}), 9.56$ (s, $2 \mathrm{H})$.

${ }^{13} \mathrm{C}$ NMR $\left(75 \mathrm{MHz}\right.$, DMSO- $d_{6}$ ): $\delta=24.4,28.6,45.7,112.2,122.4,124.0$, 146.9, 152.4, 154.9, 178.4 .

HRMS (ESI): $m / z[M+H]^{+}$calcd for $\mathrm{C}_{19} \mathrm{H}_{19} \mathrm{~N}_{6} \mathrm{O}_{4}$ : 395.1462; found: 395.1461.

5,5'-\{[1,3-Phenylenebis(1H-1,2,3-triazole-4,1-diyl)]bis(methylene)\}bis(furfural) (4q)

Yield: $229 \mathrm{mg}$ (89\%); pale yellow crystals; $\mathrm{mp} 136-139^{\circ} \mathrm{C}$. IR (KBr): 3449, 3122, 1685, 1655, 1524, 1193, 1025, $787 \mathrm{~cm}^{-1}$.

${ }^{1} \mathrm{H}$ NMR (300 MHz, DMSO- $d_{6}$ ): $\delta=5.88(\mathrm{~s}, 4 \mathrm{H}), 6.86(\mathrm{~d}, J=3.5 \mathrm{~Hz}, 2$ H), 7.50-7.56 (m, $3 \mathrm{H}), 7.84$ (dd, J = 7.7, $1.2 \mathrm{~Hz}, 2 \mathrm{H}), 8.37$ (s, $1 \mathrm{H}), 8.73$ (s, $2 \mathrm{H}), 9.58(\mathrm{~s}, 2 \mathrm{H})$.

${ }^{13} \mathrm{C}$ NMR $\left(75 \mathrm{MHz}\right.$, DMSO- $\left.d_{6}\right): \delta=46.1,112.5,121.9,122.0,123.9$, 124.8, 129.6, 131.0, 146.4, 152.5, 154.4, 178.4.

HRMS (ESI): $m / z[M+H]^{+}$calcd for $\mathrm{C}_{22} \mathrm{H}_{17} \mathrm{~N}_{6} \mathrm{O}_{4}$ : 429.1306; found: 429.1303 .

\section{2-Azidomethyl-5-ethynylfuran (AMEF) (6)}

From 2-hydroxymethyl-5-ethynylfuran (HMEF) (5): Diphenylphosphoryl azide $(0.84 \mathrm{~g}, 3.0 \mathrm{mmol})$ was added under an argon atmosphere to an ice-cold solution of 2-hydroxymethyl-5-ethynylfuran (5) $(0.25 \mathrm{~g}, 2.0 \mathrm{mmol})$ in acetonitrile $(10 \mathrm{~mL})$, followed by the addition of DBU $(0.45 \mathrm{~mL}, 3.0 \mathrm{mmol})$. The reaction mixture was stirred for $10 \mathrm{~h}$ and concentrated under vacuum. The residue was diluted with water $(10 \mathrm{~mL})$ and dichloromethane $(10 \mathrm{~mL})$ and acidified with dilute hydrochloric acid. The aqueous layer was separated and extracted with dichloromethane (2 to $10 \mathrm{~mL}$ ). The organic fractions were combined, dried over anhydrous magnesium sulfate and evaporated at $30^{\circ} \mathrm{C}$. The residue was purified by column chromatography on silica gel to afford 6 as a yellow liquid $(0.26 \mathrm{~g}, 86 \%) . R_{f}=0.45$ (hexanes/EtOAc, $4: 1$ ).

From 5-(azidomethyl)furfural (AMF) (3): Potassium carbonate (1.8 g, $13.2 \mathrm{mmol}$ ) was placed in a $100 \mathrm{~mL}$ Schlenk flask. A vacuum was applied and then the flask was repeatedly flushed with argon. A solution of 5-(azidomethyl)furfural (3) (1.0 g, $6.6 \mathrm{mmol})$ in absolute methanol $(50 \mathrm{~mL})$ was added, the mixture was stirred under an argon atmosphere for $10 \mathrm{~min}$ at room temperature and then Bestmann-Ohira reagent $(1.2 \mathrm{~mL}, 7.9 \mathrm{mmol}$ ) was added dropwise. The mixture was stirred overnight at room temperature, diluted with dichloromethane $(100 \mathrm{~mL})$, washed with water $(100 \mathrm{~mL})$ and brine $(2 \times 50 \mathrm{~mL})$, and dried over anhydrous magnesium sulfate. The solvent was removed at $30{ }^{\circ} \mathrm{C}$ under reduced pressure and the residue was purified by column chromatography on silica gel to afford 6 as a yellow liquid $(0.40 \mathrm{~g}$, $41 \%) \cdot R_{f}=0.45$ (hexanes/EtOAc, $4: 1$ ).

IR (KBr): 3290, 3127, 2098, 1404, 1203, 1019, $798 \mathrm{~cm}^{-1}$. 
${ }^{1} \mathrm{H}$ NMR $\left(300 \mathrm{MHz}, \mathrm{CDCl}_{3}\right): \delta=3.41(\mathrm{~s}, 1 \mathrm{H}), 4.28(\mathrm{~s}, 2 \mathrm{H}), 6.34(\mathrm{~d}, J=$ $3.4 \mathrm{~Hz}, 1 \mathrm{H}), 6.62(\mathrm{~d}, J=3.4 \mathrm{~Hz}, 1 \mathrm{H})$.

${ }^{13} \mathrm{C}$ NMR $\left(75 \mathrm{MHz}, \mathrm{CDCl}_{3}\right): \delta=47.1,73.7,82.5,110.4,117.1,137.0$, 150.5 .

HRMS (ESI): $m / z$ [M $+\mathrm{H}]^{+}$calcd for $\mathrm{C}_{7} \mathrm{H}_{6} \mathrm{~N}_{3} \mathrm{O}$ : 148.0505; found: 148.0511 .

\section{Synthesis of Oligomers from 2-Azidomethyl-5-ethynylfuran (6)}

With dimethylformamide as the solvent: Copper(I) bromide $(7.2 \mathrm{mg}$, $0.05 \mathrm{mmol})$ in an $8 \mathrm{~mL}$ glass vial was dissolved in DMF $(2 \mathrm{~mL})$ under an argon atmosphere. The corresponding ligand $(0.10 \mathrm{mmol})$ was added and the mixture was stirred for $5 \mathrm{~min}$. 2-Azidomethyl-5ethynylfuran (6) $(74.0 \mathrm{mg}, 0.50 \mathrm{mmol})$ was added and the mixture was stirred overnight at room temperature. The oligomer was precipitated by addition of methanol $(7 \mathrm{~mL})$, filtered, washed with diethyl ether and dried under vacuum. The yield ranged from $76-91 \%$ depending on the ligand used.

With aqueous ethanol as the solvent: Catalytic amounts of copper sulfate pentahydrate $(20.4 \mathrm{mg}, 0.08 \mathrm{mmol})$ and sodium ascorbate $(20.2$ $\mathrm{mg}, 0.10 \mathrm{mmol}$ ) were added to a solution of 2-azidomethyl-5ethynylfuran (6) (100.0 $\mathrm{mg}, 0.68 \mathrm{mmol}$ ) in $50 \%$ aqueous ethanol (10 $\mathrm{mL}$ ) and the mixture was stirred overnight. The precipitate that formed was filtered, washed with $50 \%$ aqueous ethanol and ethyl acetate and then dried in a desiccator over phosphorus pentoxide. The product weight was $70.3 \mathrm{mg}(70 \%)$.

IR (KBr): 3424, 3144, 1655, 1449, 1343, 1229, 1047, $769 \mathrm{~cm}^{-1}$.

${ }^{1} \mathrm{H}$ NMR $\left(300 \mathrm{MHz}\right.$, DMSO- $\left.d_{6}\right): \delta=5.68-5.75(\mathrm{~m}, 2 \mathrm{H}), 6.67-6.71(\mathrm{~m}, 1$ $\mathrm{H}), 6.76-6.79(\mathrm{~m}, 1 \mathrm{H}), 8.37-8.44(\mathrm{~m}, 1 \mathrm{H})$.

\section{Funding Information}

This study was supported by a Russian Science Foundation 17-1301176 grant.

\section{Acknowledgment}

We thank Dr. Alexey S. Kashin for FE-SEM characterization, Andrey M. Tsedilin and Ekaterina A. Denisova for ESI-MS spectra, Artem N. Fakhrutdinov for low-temperature NMR studies and Irina A. Borisova for IR services.

\section{Supporting Information}

Supporting information for this article is available online at https://doi.org/10.1055/s-0037-1610414.

\section{References}

(1) (a) van Putten, R. J.; van der Waal, J. C.; de Jong, E.; Rasrendra, C. B.; Heeres, H. J.; de Vries, J. G. Chem. Rev. 2013, 113, 1499. (b) Kucherov, F. A.; Romashov, L. V.; Galkin, K. I.; Ananikov, V. P. ACS Sustainable Chem. Eng. 2018, 6, 8064. (c) Sheldon, R. A. Green Chem. 2014, 16, 950. (d) Teong, S. P.; Yi, G.; Zhang, Y. Green Chem. 2014, 16, 2015. (e) Wang, T.; Nolte, M. W.; Shanks, B. H. Green Chem. 2014, 16, 548. (f) Delidovich, I.; Leonhard, K.;
Palkovits, R. Energy Environ. Sci. 2014, 7, 2803. (g) Besson, M.; Gallezot, P.; Pinel, C. Chem. Rev. 2013, 114, 1827. (h) Yu, I. K. M.; Tsang, D. C. W. Bioresour. Technol. 2017, 238, 716.

(2) Tong, X.; Ma, Y.; Li, Y. Appl. Catal., A 2010, 385, 1.

(3) (a) Roman-Leshkov, Y.; Barrett, C. J.; Liu, Z. Y.; Dumesic, J. A. Nature 2007, 447, 982. (b) Saha, B.; Bohn, C. M.; Abu-Omar, M. M. ChemSusChem 2014, 7, 3095. (c) Hu, L.; Zhao, G.; Hao, W.; Tang, X.; Sun, Y.; Lin, L.; Liu, S. RSC Adv. 2012, 2, 11184. (d) Yang, P.; Xia, Q.; Liu, X.; Wang, Y. Fuel 2017, 187, 159.

(4) (a) Delidovich, I.; Hausoul, P. J.; Deng, L.; Pfutzenreuter, R.; Rose, M.; Palkovits, R. Chem. Rev. 2016, 116, 1540. (b) Davis, M. E. Top. Catal. 2015, 58, 405. (c) Papageorgiou, G. Z.; Papageorgiou, D. G.; Terzopoulou, Z.; Bikiaris, D. N. Eur. Polym. J. 2016, 83, 202. (d) Kucherov, F. A.; Gordeev, E. G.; Kashin, A. S.; Ananikov, V. P. Angew. Chem. Int. Ed. 2017, 56, 15931.

(5) (a) Nakagawa, Y.; Tamura, M.; Tomishige, K. ACS Catal. 2013, 3 , 2655. (b) Herbst, A.; Janiak, C. CrystEngComm 2017, 19, 4092. (c) Rojas-Buzo, S.; García-García, P.; Corma, A. Green Chem. 2018, 20, 3081. (d) Fang, R.; Tian, P.; Yang, X.; Luque, R.; Li, Y. Chem. Sci. 2018, 9, 1854. (e) Romashov, L. V.; Ananikov, V. P. Org. Biomol. Chem. 2016, 14, 10593.

(6) A literature survey was carried out using SciFinder and Reaxys (November 2018).

(7) Huisgen, R. Angew. Chem., Int. Ed. Engl. 1963, 2, 565.

(8) (a) Rostovtsev, V. V.; Green, L. G.; Fokin, V. V.; Sharpless, K. B. Angew. Chem. Int. Ed. 2002, 41, 2596. (b) Kolb, H. C.; Finn, M. G.; Sharpless, K. B. Angew. Chem. Int. Ed. 2001, 40, 2004.

(9) (a) Hawker, C. J.; Wooley, K. L. Science 2005, 309, 1200. (b) Oakdale, J. S.; Kwisnek, L.; Fokin, V. V. Macromolecules 2016, 49, 4473.

(10) (a) Kolb, H. C.; Sharpless, K. B. Drug Discovery Today 2003, 8 , 1128. (b) Wang, Q.; Chan, T. R.; Hilgraf, R.; Fokin, V. V.; Sharpless, K. B.; Finn, M. G. J. Am. Chem. Soc. 2003, 125, 3192.

(11) (a) Moses, J. E.; Moorhouse, A. D. Chem. Soc. Rev. 2007, 36, 1249. (b) Topchiy, M.; Ageshina, A.; Gribanov, P.; Masoud, S.; Akmalov, T.; Nefedov, S.; Osipov, S.; Nechaev, M.; Asachenko, A. Eur. J. Org. Chem. 2018, in press; DOI: 10.1002/ejoc.201801538.

(12) Romashov, L. V.; Ananikov, V. P. Chem. Asian J. 2017, 12, 2652.

(13) Dunbabin, A.; Subrizi, F.; Ward, J. M.; Sheppard, T. D.; Hailes, H. C. Green Chem. 2017, 19, 397.

(14) For example, see: (a) Pruet, J. M.; Saito, R.; Manzano, L. A.; Jasheway, K. R.; Wiget, P. A.; Kamat, I.; Anslyn, E. V.; Robertus, J. D. ACS Med. Chem. Lett. 2012, 3, 588. (b) Goswami, P. P.; Suding, V. P.; Carlson, A. S.; Topczewski, J. J. Eur. J. Org. Chem. 2016, 4805. (c) Pramanik, S.; Ghorai, P. RSC Adv. 2013, 3, 23157. (d) Kazancioglu, E. A.; Kazancioglu, M. Z.; Fistikci, M.; Secen, H.; Altundas, R. Org. Lett. 2013, 15, 4790. (e) Padwa, A.; Crawford, K. R.; Rashatasakhon, P.; Rose, M. J. Org. Chem. 2003, 68, 2609. (f) Fabbro, C.; Armani, S.; Carloni, L.-E.; De Leo, F.; Wouters, J.; Bonifazi, D. Eur. J. Org. Chem. 2014, 5487. (g) Yakushijin, K.; Suzuki, R.; Kawaguchi, N.; Tsuboi, Y.; Furukawa, H. Chem. Pharm. Bull. 1986, 34, 2049. (h) Mascal, M.; Dutta, S. Green Chem. 2011, 13, 40. (i) Saikachi, H.; Kitagawa, T. Chem. Pharm. Bull. 1978, 26, 1054.

(15) (a) Nishida, M.; Fujii, S.; Aoki, T.; Hayakawa, Y.; Muramatsu, H.; Morita, T. J. Fluorine Chem. 1990, 46, 445. (b) Dominguez, C.; Csaky, A. G.; Plumet, J.; Rigal, L.; Tauler, C. Synth. Commun. 1991, 21, 1251. (c) Carpita, A.; Rossi, R.; Veracini, C. A. Tetrahedron 1985, 41, 1919.

(16) (a) Rogers, S. A.; Melander, C. Angew. Chem. Int. Ed. 2008, 47, 5229. (b) Kim, W. J.; Korthals, K. A.; Li, S.; Le, C.; Kalisiak, J.; Sharpless, K. B.; Fokin, V. V.; Miyamoto, Y.; Eckmann, L. Antimicrob. Agents Chemother. 2017, 61, e02397-16. (c) Ciardiello, J. J.; 
Stewart, H. L.; Sore, H. F.; Galloway, W.; Spring, D. R. Bioorg. Med. Chem. 2017, 25, 2825. (d) Menendez, C.; Rodriguez, F.; Ribeiro, A. L.; Zara, F.; Frongia, C.; Lobjois, V.; Saffon, N.; Pasca, M. R.; Lherbet, C.; Baltas, M. Eur. J. Med. Chem. 2013, 69, 167. (e) Menendez, C.; Gau, S.; Ladeira, S.; Lherbet, C.; Baltas, M. Eur. J. Org. Chem. 2012, 409. (f) Nawratil, S.; Grypioti, M.; Menendez, C.; Mallet-Ladeira, S.; Lherbet, C.; Baltas, M. Eur. J. Org. Chem. 2014, 654. (g) Kumar, R.; Verma, D.; Mobin, S. M.; Namboothiri, I. N. Org. Lett. 2012, 14, 4070. (h) Yamamoto, K.; Bruun, T.; Kim, J. Y.; Zhang, L.; Lautens, M. Org. Lett. 2016, 18, 2644. (i) Kudryavtseva, T. N.; Lamanov, A. Y.; Klimova, L. G.; Nazarov, G. V. Russ. J. Gen. Chem. 2018, 88, 676.

(17) Zhang, S.; Xu, Z.; Gao, C.; Ren, Q. C.; Chang, L.; Lv, Z. S.; Feng, L. S. Eur. J. Med. Chem. 2017, 138, 501.

(18) (a) Kalhor-Monfared, S.; Beauvineau, C.; Scherman, D.; Girard, C. Eur. J. Med. Chem. 2016, 122, 436. (b) Dadmal, T. L.; Appalanaidu, K.; Kumbhare, R. M.; Mondal, T.; Ramaiah, M. J.; Bhadra, M. P. New J. Chem. 2018, 15546.
(19) Polymer Chemistry Series: Click Polymerization; Qin, A.; Tang, B. Z., Ed.; The Royal Society of Chemistry: Cambridge, 2018.

(20) Galkin, K. I.; Krivodaeva, E. A.; Romashov, L. V.; Zalesskiy, S. S.; Kachala, V. V.; Burykina, J. V.; Ananikov, V. P. Angew. Chem. Int. Ed. 2016, 55, 8338 .

(21) Mascal, M.; Nikitin, E. B. Angew. Chem. Int. Ed. 2008, 47, 7924.

(22) Armarego, W. L. F. Purification of Laboratory Chemicals; Butterworth-Heinemann: Oxford, 2017, 8th ed.

(23) Molander, G. A.; Cadoret, F. Tetrahedron Lett. 2011, 52, 2199.

(24) Shi, E.; Pei, C. Synthesis 2004, 2995.

(25) Wijtmans, M.; de Graaf, C.; de Kloe, G.; Istyastono, E. P.; Smit, J.; Lim, H.; Boonnak, R.; Nijmeijer, S.; Smits, R. A.; Jongejan, A.; Zuiderveld, O.; de Esch, I. J. P.; Leurs, R. J. Med. Chem. 2011, 54, 1693. 